\title{
Fixed-Point Solution of Plant Input/Location Problems*
}

\author{
A. J. Goldman \\ National Bureau of Standards, Institute for Basic Standards, Washington, D.C. 20234 \\ (March 14, 1974) \\ This paper considers the following generalization of the Weber plant location problem: the plant's \\ output level is fixed, and its levels of input from its supply points, as well as its location, are among \\ the decision variables. Hurter and Wendell (J. Reg. Sci., 1972) showed that this problem admits a \\ kind of separability when the plant's production function lies in a certain class including the Cobb- \\ Douglas forms. The present paper (a) determines the extent of that function-class, (b) carries out the \\ explicit separation for the CES generalization of the Cobb-Douglas functions, and (c) discusses simple \\ fixed-point-type iterative solution algorithms, similar to that well-known for the ordinary Weber problem, \\ for several production functions (Cobb-Douglas, CES, and various two-stage technologies). Local \\ convergence of these algorithms is established; computational experience will be reported in a separate \\ Part II.
}

Key words: CES; economics; Leontief; location theory; plant location; production functions; transportation; Weber problem, mathematical programming.

\section{Introduction}

The "ordinary" Weber plant-location problem, set in the real $n$-dimensional space $R$ ", can be described as requiring the selection of $x \in R^{n}$ to minimize the function

$$
\phi_{W}(x)=\sum_{1}^{m} t_{i}|| x-s_{i}|| q_{i}
$$

Here the decision variable $x$ represents the location of a plant which requires $m$ inputs for its operation; $s_{i}$ is the source of the $i$ th input, $t_{i}$ the associated unit transportation cost, and $q_{i}$ the level of the $i$ th input. In (1.1), $\|\cdot\|$ denotes some appropriate norm on $R^{n}$, which will be taken as the Euclidean norm throughout. The abbreviation

$$
\rho_{i}=\left\|x-s_{i}\right\|
$$

will prove convenient.

This problem can be generalized by including the vector $q=\left(q_{1}, \ldots, q_{m}\right)$ of input levels among the decision variables. A plant output level $q^{0}$ is specified, as is the plant's production function $f(q)$ and the unit prices $p_{i}$ of the inputs at their respective sources $s_{i}$. Now the problem is to choose $x \in R^{n}$ and $q \epsilon R^{m}$ so as to achieve

$$
\min _{x} \min _{q} \sum_{1}^{m}\left\{t_{i} \rho_{i}+p_{i}\right\} q_{i}
$$

subject to

$$
f(q)=q^{0} .
$$

AMS Classification: Primary 90B30; Secondary 90A99, 90C30, 90C50.

*Affectionally dedicated to Professor A. W. Tucker on the occasion of his retirement from the Department of Mathematics. Princeton University. 
A one-dimensional version of this problem was studied by Sakashita $[1]^{1}$ and extended to a network location problem by Wendell and Hurter [2]. The general case was formulated by Wendell and Hurter in a paper [3] which is the point of departure for the present work.

The production function $f$ will be said to lie in "class $C\left(q^{0}\right)^{\text {" }}$ if there is a positive constant $K\left(q^{0}\right)$ such that, for all positive $q$ satisfying (1.4),

$$
\sum_{1}^{m} q_{i} \partial f / \partial q_{i}=K\left(q^{0}\right)
$$

It is noted in [3] that for this class of production functions, the problem has a kind of separability: it can be transformed into

$$
\min _{x} \mu(x)
$$

where $\mu(x)$ is the Lagrange multiplier corresponding to constraint (1.4) for the "inner" minimization in (1.3).

If $f$ lies in class $C\left(q^{0}\right)$ for all $q^{0}>0$, we say it lies in "class $C$ ". As noted in [3], it is a consequence of Euler's theorem that class $C$ contains all differentiable homogeneous functions, but it contains other functions as well. In section 2, we determine the extent of class $C$.

Since class $C$ contains the homogeneous production functions, it in particular includes the familiar Cobb-Douglas functions [4]

$$
f(q)=\exp \left\{a+\sum_{1}^{m} a_{i} \log q_{i}\right\} \quad\left(a_{i}>0\right)
$$

as well as the multi-input CES ("constant elasticity of substitution") functions of Arrow, Chenery, Minhas and Solow [5],

$$
f(q)=\left(\sum_{1}^{m} b_{i} q_{i}^{-c}\right)^{-1 / c} \quad\left(b_{i}>0, c>-1, c \neq 0\right) .
$$

In [3] the separation (1.6) is carried out explicitly for the Cobb-Douglas case, but the corresponding problem for other homogeneous functions is noted to be "difficult". In section 3 , we perform the explicit separation for the CES functions and for several functions representing two-stage technologies.

The ordinary Weber problem can be regarded as arising from a Leontief production function (in which specifying the output-level $q^{0}$ fixes the values $q_{i}$ ), and is a convex programming problem. In contrast, the mathematical programming problems (1.6) arising from the cases studied here are nonconvex, so that their numerical solution is nontrivial. In section 4 , simple iterative fixed-pointtype solution algorithms are presented, patterned after one well known for the Weber problem. Local convergence is established for the low dimensions of practical interest, and the analyses necessary to handle the singularities $s_{i}$ are performed. A subsequent Part II will report our computational experience, to date, with these algorithms. Further work should take up the case of timevarying prices $p_{i}$, transport costs $t_{i}$, and output levels $q^{0}$. Another natural line of generalization would incorporate consideration of market location.

\section{Determination of Class $C$}

From (1.4) and (1.5), it is readily seen that $C$ is the class of production functions $f$ which satisfy a partial differential equation of the form

$$
\sum_{1}^{m} q_{i} \partial f / \partial q_{i}=F[f(q)]
$$

\footnotetext{
${ }^{1}$ Figures in brackets indicate the literature references at the end of this paper.
} 
for all positive $q$. Since a production function $f$ has positive first-order derivatives, $F$ is positivevalued; we also assume it continuous.

THEOREM: Class C consists of the production functions of the form $\mathrm{f}(\mathrm{q})=\mathrm{M}[\mathrm{h}(\mathrm{q})]$, where $\mathrm{h}$ is a production function homogeneous of degree 1 , and $\mathrm{M}$ is an increasing differentiable function.

Before proving this theorem, we note three consequences of it. First, it identifies class $C$ with the class of "homothetic" production functions introduced by Shephard (see p. 30 of [6]), apart from questions of smoothness and other properties in a theoretical definition of "production function". Second, it "explains" the examples of nonhomogeneous members of $C$ given in [3], which in fact are the logarithms of Cobb-Douglas functions. Third, it implies that analyses of our generalized Weber problem can be confined to production functions which are homogeneous of degree 1 , since with $f=M[h]$ as above, the constraint (1.4) can be replaced by the equivalent $h(q)=M^{-1}\left(q^{0}\right)$.

For the proof, first assume $f=M[h]$ as in the theorem's statement. By Euler's theorem on homogeneous functions,

$$
\sum_{1}^{m} q_{i} \partial h / \partial q_{i}=h(q)
$$

and so by the chain rule of differentiation

$$
\sum_{1}^{m} q_{i} \partial f / \partial q_{i}=M^{\prime}[h(q)] h(q),
$$

an instance of $(2.1)$ with $F(v)=M^{\prime}\left[M^{-1}(v)\right] M^{-1}(v)$.

Conversely, suppose $f \in C$ satisfies (2.1). Define a function $M^{-1}(u)$ by

$$
M^{-1}(u)=\exp \left\{\int_{u_{0}}^{u}[1 / F(v)] d v\right\}
$$

for some $u_{0}>0$. Since $F$ is positive-valued and continuous, $M^{-1}$ is increasing and differentiable, and thus has an increasing differentiable inverse function $M$. Define $h=M^{-1}[f]$; then by (2.1) and $(2.2)$

$$
\begin{aligned}
\sum_{1}^{m} q_{i} \partial h / \partial q_{i} & =\left(M^{-1}\right)^{\prime}[f] \sum_{1}^{m} q_{i} \partial f / \partial q_{i} \\
& =\left\{M^{-1}[f] / F[f]\right\} F[f]=h,
\end{aligned}
$$

so that the converse of Euler's theorem implies that $h$ is homogeneous of degree 1. Since $f=M[h]$, the proof is complete.

Before leaving this topic, we note that class $C$ also arises in a multi-output generalization of the problem under discussion. Namely, suppose the $m$ inputs are used jointly to produce several outputs in accordance with a vector production function having one component $f_{j}(q)$ per output. Suppose furthermore that the level of each output is prescribed, and that $\mu_{j}(x)$ denotes the Lagrange multiplier corresponding to the $j$ th of these constraints in the inner minimization of (1.3). The first-order optimality conditions for that minimization are

$$
t_{i} \rho_{i}+p_{i}=\sum_{j} \mu_{j}(x) \partial f_{j} / \partial q_{i} \quad(i=1,2, \ldots, m),
$$

so that with the inner minimization accomplished for each $x$ (and the $\partial f_{j} / \partial q_{i}$ evaluated at its solution) the objective function (1.2) becomes

$$
\sum_{i}\left(t_{i} \rho_{i}+p_{i}\right) q_{i}=\sum_{j} \mu_{j}(x) \sum_{i} q_{i} \partial f_{j} / \partial q_{i \cdot}
$$


If now each $f_{j}$ is in class $C$, with corresponding $F_{j}$ in $(2.1)$, then this minimand is equal to

$$
\phi(x)=\sum_{j} \mu_{j}(x) F_{j}\left(f_{j}^{0}\right)
$$

where $f_{j}^{0}$ is the prescribed level of the $j$ th output. Result (2.3) is a multi-output extension of the separability expressed by (1.6). This (multiple output) line of generalization will not be pursued further in the present paper.

\section{Some Cases of Explicit Separation}

To take full advantage of the separability expressed in (1.6), it is necessary to find an explicit expression for $\mu(x)$, so that the resultant "pure location" problem is in explicit form. This will be possible, in particular, if $f$ satisfies a suitable set of identities

$$
q_{i}=F_{i}\left[f(q), \partial f / \partial q_{i}\right]
$$

To see why this is so, recall the first-order optimality conditions

$$
t_{i} \rho_{i}+p_{i}=\mu(x) \partial f / \partial q_{i}
$$

for the inner minimization in (1.3). The solution $q(x)$ of that minimization will therefore satisfy, by (3.1),

$$
q_{i}(x)=G_{i}\left[\left(t_{i} \rho_{i}+p_{i}\right) / \mu(x)\right]
$$

where $G_{i}(\cdot)=F_{i}\left(q^{0}, \cdot\right)$. It follows that

$$
q^{0}=f[q(x)]=f\left\{G_{1}\left[\left(t_{1} \rho_{1}+p_{1}\right) / \mu(x)\right], \ldots, G_{m}\left[\left(t_{m} \rho_{m}+p_{m}\right) / \mu(x)\right]\right\} .
$$

Typically (hence the adjective "suitable" above (3.1)) this equation can be solved to obtain the desired explicit form for $\mu(x)$.

For the Cobb-Douglas case (1.7), one has in (3.1)

$$
F_{i}(u, v)=a_{i} u / v
$$

thus (3.2) yields

$$
\begin{aligned}
\log q^{0} & =a+\sum_{1}^{m} a_{i} \log \left[a_{i} q^{0} \mu(x) /\left(t_{i} \rho_{i}+p_{i}\right)\right] \\
& =\left[a+\sum_{1}^{m} a_{i} \log \left(a_{i} q^{0}\right)\right]+[\log \mu(x)] \sum_{1}^{m} a_{i}-\sum_{1}^{m} a_{i} \log \left(t_{i} \rho_{i}+p_{i}\right) .
\end{aligned}
$$

It follows that

$$
\log \mu(x)=\left(\sum_{1}^{m} a_{i}\right)^{-1} \sum_{1}^{m} a_{i} \log \left(t_{i} \rho_{i}+p_{i}\right)+\text { const. }
$$

or, with the abbreviation

$$
\begin{gathered}
\pi_{i}=p_{i} / t_{i} \\
\log \mu(x)=\left(\sum_{1}^{m} a_{i}\right)^{-1} \sum_{1}^{m} a_{i} \log \left(\rho_{i}+\pi_{i}\right)+\text { const. }
\end{gathered}
$$


Thus the pure location problem (1.6) is equivalent in the Cobb-Douglas case to minimizing

$$
\phi_{C D}(x)=\sum_{1}^{m} a_{i} \log \left(\rho_{i}+\pi_{i}\right)
$$

a result derived in [3].

For the CES production function (1.8), one has in (3.1)

$$
F_{i}(u, v)=u\left(b_{i} / v\right)^{1 /(c+1)} .
$$

With $d=c /(c+1),(3.2)$ yields

$$
\begin{aligned}
\left(q^{0}\right)^{-c} & =\sum_{1}^{m} b_{i}\left[q^{0}\left(b_{i} \mu(x) /\left\{t_{i} \rho_{i}+p_{i}\right\}\right)^{1 /(c+1)}\right]^{-c} \\
& =\left(q^{0}\right)^{-c}[\mu(x)]^{-d} \sum_{1}^{m} b_{i}^{1-d}\left\{t_{i} \rho_{i}+p_{i}\right\}^{d} \\
& =\left(q^{0}\right)^{-c}[\mu(x)]^{-d} \sum_{1}^{m} c_{i}\left\{\rho_{i}+\pi_{i}\right\}^{d}
\end{aligned}
$$

where $\pi_{i}$ is as above and

$$
c_{i}=b_{i}^{1-d} t_{i}^{d}
$$

If $c>0$, so that $0<d<1$, then minimizing $\mu(x)$ is equivalent to minimizing $[\mu(x)]^{d}$, and it follows that the pure location problem for the CES case can be expressed as demanding the minimization of

$$
\phi_{C E S}(x)=\sum_{1}^{m} c_{i}\left\{\rho_{i}+\pi_{i}\right\}^{d} \quad(0<d<1) .
$$

If $d<0$ (i.e., $-1<c<0$ ), the pure location problem involves maximizing the form (3.6), or equivalently minimizing its negative. Our subsequent discussion of the CES situation is readily adapted to this subcase, but will for simplicity be confined to the subcase $0<d<1$; the reader is warned that the later discussion does not as it stands refer to the case $c<0$ (i.e., $d<0$ ), though the revision is simple.

The three functions $\phi_{W}, \phi_{C D}, \phi_{C E S}$ all have the form

$$
\phi(x)=\sum_{1}^{m} \phi_{i}\left(\rho_{i}\right)
$$

where the functions $\phi_{i}$ are defined and nonnegative on $(0, \infty)$, positive-valued and twice differentiable on $(0, \infty)$, and satisfy

$$
\phi_{i}^{\prime}>0 \quad \text { on }(0, \infty) \text {. }
$$

But while for $\phi_{W}$, which has $\phi_{i}(u)=\left(t_{i} q_{i}\right) u$, each summand in (3.7) is a convex function of $x$, neither $\phi_{C D}$ nor $\phi_{C E S}$ is convex, so that the $C D$ and $C E S$ cases give rise to nonconvex programming problems. This nonconvexity is most easily seen in the one-dimensional case; whereas $\phi_{W}$ is linear on each (open) interval between successive points $s_{i}$, both $\phi_{C D}$ and $\phi_{C E S}$ satisfy $\phi^{\prime \prime}<0$ (the antithesis of convexity) on those intervals.

The absence of convexity suggests the possibility of multiple local minima, and these can in fact occur. They may occur at a point $s_{i}$ (in the one-dimensional case, local minima occur only at points $s_{i}$ ), which however would not be routinely identified as a critical point since $s_{i}$ is a singular point of 


$$
\operatorname{grad} \rho_{i}=\left(x-s_{i}\right) / \rho_{i}
$$

and thus of $\operatorname{grad} \phi$. We therefore proceed to develop a special test for the existence of a local minimum at an $s_{i}$, say $s_{1}$. It will be assumed that

$$
s_{i}=s_{1} \quad \text { for } 1 \leqslant i \leqslant r, \quad s_{i} \neq s_{1} \quad \text { for } i>r .
$$

Let $\theta$ be a nonnegative scalar variable, and $w \epsilon R^{n}$ be a variable "direction vector", i.e., $\|w\|$ $=1$. Set

$$
g(\theta, w)=\phi\left(s_{1}+\theta w\right) .
$$

Then a necessary condition for a local minimum at $s_{1}$ is that

$$
\inf _{w} \partial g(0+, w) / \partial \theta \geqslant 0
$$

holds. For $x=s_{1}+\theta w$, one has

$$
\rho_{i}=\theta \quad \text { for } i \leqslant r, \quad \rho_{i}=\left\|\theta w-\left(s_{i}-s_{1}\right)\right\| \quad \text { for } i>r,
$$

so that

$$
\begin{gathered}
g(\theta, w)=\sum_{1}^{r} \phi_{i}(\theta)+\sum_{r+1}^{m} \phi_{i}\left(\left\|\theta w-\left(s_{i}-s_{1}\right)\right\|\right), \\
\partial g / \partial \theta=\sum_{1}^{r} \phi_{i}^{\prime}(\theta)+\sum_{r+1}^{m}\left[\phi_{i}^{\prime}\left(\rho_{i}\right) / \rho_{i}\right]\left[6-\left(w, s_{i}-s_{1}\right)\right]
\end{gathered}
$$

where $\left(w, s_{i}-s_{1}\right)$ denotes the scalar product. With the notations

$$
\begin{gathered}
b=\sum_{r+1}^{m}\left[\phi_{i}^{\prime}\left(\left\|s_{i}-s_{1}\right\|\right) /\left\|s_{i}-s_{1}\right\|\right]\left(s_{i}-s_{1}\right), \\
A=\sum_{1}^{r} \phi_{i}^{\prime}(0),
\end{gathered}
$$

it follows from (3.11) that

$$
\partial g(0+, w) / \partial \theta=A-(w, b) .
$$

The Cauchy-Schwarz inequality implies

$$
(w, b) \leqslant\|b\|,
$$

with equality for all $w$ if $b=0$, and for $w=b /\|b\|$ and its negative if $b \neq 0$. It follows from (3.14) that

$$
\min _{w} \partial g(0+, w) \partial \theta=A-\|b\|,
$$

and so

$$
A \geqslant\|b\|
$$

is a necessary condition for a local minimum at $s_{1}$.

Conversely, suppose

$$
\sigma=A-\|b\|>0
$$

Choose any positive $\delta_{1}<\min _{i>r}\left\|s_{i}-s_{i}\right\|$. Then the right-hand side of (3.11), call it $g^{*}(\theta, w)$, 
is continuous on the compact domain $\left[0, \delta_{1}\right] \times\{w:\|w\|=1\}$, hence uniformly continuous. In particular there is a $\delta>0$, with $\delta \leqslant \delta_{1}$, such that if $0 \leqslant \theta \leqslant \delta$ then for all $w$

$$
g^{*}(\theta, w)>g^{*}(0, w)-\sigma .
$$

Since the previous analysis implies $g^{*}(0, w) \geqslant \sigma$, we have demonstrated the existence of a $\delta>0$ such that

$$
\partial g(\theta, w) / \partial \theta=g^{*}(\theta, w)>0 \quad \text { for } 0 \leqslant \theta \leqslant \delta \text { and all } w .
$$

Thus in the $\delta$-neighborhood of $s_{1}, \phi$ is uniquely minimized at the point $s_{1}$, so that (3.16) is a sufficient condition for a strict local minimum at $s_{1}$. It is a generalization of its specialization (given by Kuhn and Kuenne [7]) for the ordinary Weber problem.

Next we consider some cases in which the "inputs" transported to the plant from the sourcepoints $s_{i}$ are best interpreted as "factors of production", not for the process yielding the plant's final output, but rather for intermediate on-site processes producing these "final factors." Note that the production functions for these intermediate processes, as well as that for the final process, must now be specified. The levels of the final factors are (intermediate) variables of the problem; these levels will be denoted

$$
Q=\left(Q_{1}, Q_{2}, \ldots, Q_{M}\right)
$$

and the production function for the final process will be denoted $f(Q)$.

A variety of interesting problems can be posed in this context; we will briefly take up just a few of them. For notation, it will be convenient to partition the input-indexing set $\{1,2, \ldots, m\}$ into subsets $\{I(\nu): \nu=1,2, \ldots, M\}$, where $i \epsilon I(\nu)$ signifies that the $i$ th input goes into making the $\nu$ th final factor. Assuming disjointness of these sets $I(\nu)$ is not really a restriction on the technology-so long as capacity constraints at the sources are omitted-since otherwise-identical inputs can be artifically distinguished according to the final factor in which they will be embodied.

Suppose first that each intermediate process follows a simple Leontief production law; that is, there are positive constants $K_{i}$ such that

$$
q_{i}=K_{i} Q_{\nu} \quad \text { for all } i \epsilon I(\nu)
$$

Then the problem can be written

subject to

$$
\min _{x} \min _{Q} \sum_{\nu}\left[\sum_{i \in I(\nu)}\left\{t_{i} \rho_{i}+p_{i}\right\} K_{i}\right] Q_{\nu}
$$

$$
f(Q)=q^{0}
$$

Reduction to a pure location problem follows the same pattern as before; if $f$ is a Cobb-Douglas function with parameters $a_{\nu}$, or a CES function with parameters $b_{\nu}$ and $c$, the result is an objective function

$$
\phi_{C D}^{L}(x)=\sum_{\nu} a_{\nu} \log \left(\sum_{i \in I(\nu)} L_{i}\left\{\rho_{i}+\pi_{i}\right\}\right),
$$

with $L_{i}=K_{i} t_{i}, \pi_{i}=p_{i} / t_{i}$, or

$$
\begin{aligned}
\phi_{C E S}^{L}(x) & =\sum_{\nu} b_{\nu}^{1-d}\left(\sum_{i \in I(\nu)} K_{i}\left\{t_{i} \rho_{i}+p_{i}\right\}\right)^{d} \\
& =\sum_{\nu}\left(\sum_{i \in I(\nu)} L_{i}\left\{\rho_{i}+\pi_{i}\right\}\right)^{d}
\end{aligned}
$$


with $d=c /(c+1), \pi_{i}$ as above, and $L_{i}=b_{\nu}^{1 / c} K_{i} t_{i}$.

Next suppose that the final process is of Cobb-Douglas type, with parameters $a_{\nu}$. If each intermediate process is also Cobb-Douglas, then the $q_{i}$ 's are related to the plant output by a CobbDouglas function, so that the material leading to (3.4) applies. Let us suppose, instead, that each intermediate process is of CES type; assume the $\nu$ th final factor has a CES production function with parameters $\left\{b_{i}: i \epsilon I(\nu)\right\}$ and $c_{\nu}$. The result is a (composite) production function, for the plant, of Uzawa-CES type [8],

$$
\psi(q)=\exp \left\{a-\sum_{\nu}\left(a_{\nu} / c_{\nu}\right) \log \left(\sum_{i \in I(\nu)} b_{i} q_{i}^{-c_{\nu}}\right)\right\} .
$$

It is readily verified that

$$
\partial \psi / \partial q_{i}=a_{\nu} b_{i} q^{-\left(c_{\nu}+1\right)} \psi / \sum_{j \in I(\nu)} b_{j} q_{g}^{-c_{\nu}}(i \epsilon I(\nu))
$$

Although identities of type (3.1) are lacking, the general approach can still be carried out. Let

$$
\alpha_{i}(x)=t_{i} \rho_{i}+p_{i},
$$

then (3.23) and the first-order optimality conditions below (3.1) yield

$$
\alpha_{i}=\mu a_{\nu} b_{i} q_{i}^{-\left(c_{\nu}+1\right)} \psi / \sum_{j \in I(\nu)} b_{j} q_{j}^{-c_{\nu}} \quad(i \epsilon I(\nu))
$$

or equivalently, with $d_{\nu}=c_{\nu} /\left(c_{\nu}+1\right)$,

$$
q_{i}^{-c_{\nu}}=(\mu \psi)^{-d_{\nu}}\left(\alpha_{i} / a_{\nu} b_{i}\right)^{-d_{\nu}}\left(\sum_{j \in I(\nu)} b_{j} q_{j}^{-c_{\nu}}\right)^{d_{\nu}} \quad(i \epsilon I(\nu)) .
$$

Multiply both sides by $b_{i}$ and sum over $i \epsilon I(\nu)$; the result is

$$
\sum_{i \in I(\nu)} b_{i} q_{i}^{-c_{\nu}}=\left(\mu \psi a_{\nu}\right)^{-d_{\nu}}\left(\sum_{j \in I(\nu)} b_{j} q_{j}^{-c_{\nu}}\right)^{d_{\nu}} \sum_{i \in I(\nu)} b_{i}\left(\alpha_{i} / b_{i}\right)^{d_{\nu}}
$$

or equivalently

$$
\sum_{i \in I(\nu)} b_{i} q_{i}^{-c_{\nu}}=\left(\mu \psi a_{\nu}\right)^{-c_{\nu}}\left[\sum_{i \in I(\nu)} b_{i}\left(\alpha_{i} / b_{i}\right)^{d_{\nu}}\right]^{c_{\nu}+1} .
$$

i.e.,

$$
\log \left(\sum_{i \in I(\nu)} b_{i} q_{i}^{-c_{\nu}}\right)=-c_{\nu} \log \mu-c_{\nu} \log \left(\psi a_{\nu}\right)+\left(c_{\nu}+1\right) \log \left[\sum_{i \in I(\nu)} b_{i}\left(\alpha_{i} / b_{i}\right)^{d_{\nu}}\right],
$$

leading via (3.22) to

$$
\log q^{0}=a+\left(\sum_{\nu} a_{\nu}\right) \log \mu+\sum_{\nu} a_{\nu} \log \left(q^{0} a_{\nu}\right)-\sum_{\nu}\left(a_{\nu} / d_{\nu}\right) \log \left[\sum_{i \in I(\nu)} b_{i}\left(\alpha_{i} / b_{i}\right)^{d}{ }_{\nu}\right] .
$$

Thus, with the abbreviations

$$
\beta_{\nu}=a_{\nu} / d_{\nu}, \quad \pi_{i}=p_{i} / t_{i}, L_{i}=b_{i}^{1-d_{v}} t_{i}^{d},
$$

the objective function for the pure location problem takes the form

$$
\phi_{C D}^{C E S}(x)=\sum_{\nu} \beta_{\nu} \log \left[\sum_{i \in I(\nu)} L_{i}\left\{\rho_{i}+\pi_{i}\right\}^{d_{\nu}}\right] \text {. }
$$


If the $f_{\nu}$ 's are as above, but $f$ is a $C E S$ function with parameters $b_{\nu}$ and $c$, then the composite production function is

$$
\psi(q)=\left\{\sum_{\nu} b_{\nu}\left[\sum_{i \in I(\nu)} b_{i} q_{i}{ }^{-c_{\nu}}\right] c / c_{\nu}\right\}^{-1 / c} .
$$

Manipulations like those above lead to an objective function

$$
\phi_{C E S}^{C E S}(x)=\sum_{\nu}\left[\sum_{i \in I(\nu)} L_{i}\left\{\rho_{i}+\pi_{i}\right\}^{d_{\nu}}\right] d / d_{\nu},
$$

with $d=c /(c+1), d_{\nu}=c_{\nu} /\left(c_{\nu}+1\right), \pi_{i}=p_{i} / t_{i}$ and $L_{i}=b_{\nu}^{d_{\nu} / c} b_{i}^{1-d_{\nu}} t_{i}^{d_{\nu}}$.

Now assume $f$ is as above, but the $f_{\nu}$ 's are Cobb-Douglas functions with parameters $a_{\nu}$ and $\left\{a_{i}: i \epsilon I(\nu)\right\}$. Then the composite production function is

$$
\psi(q)=\left\{\sum_{\nu} b_{\nu}\left[\exp \left(a_{\nu}+\sum_{i \in I(\nu)} a_{i} \log q_{i}\right)\right]^{-c}\right\}^{-1 / c} .
$$

For the explicit separation to be tractable, it appears necessary to require each $f_{\nu}$ to be homogeneous of the same degree, i.e.,

$$
\sum_{i \in I(\nu)} a_{i}=\Delta \quad(\text { all } \nu)
$$

With the notations

$$
\begin{aligned}
& \sigma=c /(1+c \Delta), \quad A_{\nu}=a_{\nu}+\sum_{i \in I(\nu)} a_{i} \log a_{i}, \\
& \pi_{i}=p_{i} / b_{i}, \quad a_{i}^{\prime}=\sigma a_{i} \\
& a_{\nu}^{\prime}=\sum_{i \in I(\nu)} a_{i}^{\prime} \log t_{i}+(1-\sigma \Delta) \log b_{\nu}-\sigma A_{\nu},
\end{aligned}
$$

an objective function

$$
\phi_{C E S}^{C D}(x)=\sum_{\nu} \exp \left\{a_{\nu}^{\prime}+\sum_{i \in I(\nu)} a_{i}^{\prime} \log \left(\rho_{i}+\pi_{i}\right)\right\}
$$

a sum of Cobb-Douglas functions, is obtained for the pure location problem.

The reasonableness of the restriction (3.29) is supported by the following observation, which applies to the situations (3.25), (3.27), (3.30) above. Suppose $f \epsilon C$, with associated function $F$ in (3.1), and that the functions $f_{\nu}$ are homogeneous of respective degrees $\Delta_{\nu}$. For the two-stage technology to admit the kind of analysis given in this paper, the composite production function $\psi$ must lie in $C$. Now

$$
\begin{aligned}
\sum_{i} q_{i} \partial \psi / \partial q_{i} & =\sum_{\nu} \partial f / \partial Q_{\nu} \sum_{i \in I(\nu)} q_{i} \partial f_{\nu} / \partial q_{i} \\
& =\sum_{\nu} \Delta_{\nu} Q_{\nu} \partial f / \partial Q_{\nu},
\end{aligned}
$$

and only if all $\Delta_{\nu}$ have a common value $\Delta$ can we continue to the

$$
=\Delta F[\psi]
$$

which shows that $\psi \epsilon C$.

We return now to the matter of testing for a local minimum at a point $s_{i}$. Since the objective functions (3.20), (3.21), (3.25), (3.27) and (3.30) are not of the form (3.7), the test (3.16) does not apply. Instead, these objective functions have the more general form 


$$
\phi(x)=\sum_{\nu} \phi_{\nu}\left(\rho_{\nu}\right)
$$

where $\rho_{\nu}$ is a vector with components $\left\{\rho_{i}: i \epsilon I(\nu)\right\}$. For the point $s$ being tested, define the indexsets

$$
E(\nu)=\left\{i \epsilon I(\nu): s_{i}=s\right\}, \quad U(\nu)=I(\nu)-E(\nu),
$$

and introduce a variable vector $u_{\nu}$ with components $\left\{u_{i}: i \epsilon I(\nu)\right\}$ as general argument of $\phi_{\nu}$. As in the analysis leading to (3.16), let

$$
g(\theta, w)=\phi(s+\theta w)
$$

with $\theta$ a nonnegative scalar variable and $w \epsilon R^{n}$ a direction vector. Then

$$
\begin{aligned}
\partial g / \partial \theta= & \sum_{\nu} \sum_{i \in I(\nu)}\left[\partial \phi_{\nu} / \partial u_{i}\right]\left(\left\{\left\|\theta w-\left(s_{j}-s\right)\right\|\right\}_{j \in I(\nu)}\right) \\
& {\left[\theta-\left(w, s_{i}-s\right)\right] /\left\|\theta w-\left(s_{i}-s\right)\right\| } \\
= & \sum_{\nu} \sum_{i \in E(\nu)}\left[\partial \phi_{\nu} / \partial u_{i}\right]\left[\left\{\left\|\theta w-\left(s_{j}-s\right)\right\|\right\}_{j \in I(\nu)}\right] \\
+ & \sum_{\nu} \sum_{i \in U(\nu)}\left[\partial \phi_{\nu} / \partial u_{i}\right]\left(\left\{\left\|\theta w-\left(s_{j}-s\right)\right\|\right\}_{j \in I(\nu)}\right] \\
& {\left[\theta-\left(w, s_{i}-s\right)\right] /\left\|\theta w-\left(s_{i}-s\right)\right\| . }
\end{aligned}
$$

It follows that

$$
\begin{aligned}
\partial g(0+, w) / \partial \theta= & \sum_{\nu} \sum_{i \in E(\nu)}\left[\partial \phi_{\nu} / \partial u_{i}\right]\left(\left\{\left\|s_{j}-s\right\|\right\}_{j \in I(\nu)}\right) \\
& -\sum_{\nu} \sum_{i \in U(\nu)}\left[\partial \phi_{\nu} / \partial u_{i}\right]\left(\left\{|| s_{j}-s \|\right\}_{j \in I(\nu)}\right)\left(w, s_{i}-s\right) /\left\|s_{i}-s\right\| .
\end{aligned}
$$

Arguing as in the derivation of (3.16), we obtain the criterion

where now

$$
A>\|b\|
$$

Note that in (3.33) the arguments $\left\|s_{j}-s\right\|=0$ for $j \epsilon E(\nu)$.

One might also consider a two-stage process with the final stage of Leontief type. But then fixing $q^{0}$ fixes all $Q_{\nu}$, so that the problem is equivalent to one of the single-stage multi-output type described at the end of section 2 .

The final situation to be considered is that the various inputs $i \epsilon I(\nu)$ are of the $\nu$ th final factor itself (without further processing), but are distinguished merely by being from different sources. That is, the $\nu$ th intermediate process has as "production function" the additive

$$
Q_{\nu}=\sum_{i \in I(\nu)} q_{i}
$$

Here formal use of the preceding approach would lead to nonsense. The reason lies in the reliance of that approach upon the optimality condition below (3.1) to characterize the inner minimum in (1.3). In fact, that condition is guaranteed only for those $q_{i}$ which are strictly positive at the minimum, a condition which indeed is satisfied in all the previous cases treated, but is violated here since each final factor would be purchased only from the least expensive of its sources. 
With $\alpha_{i}(x)$ defined as in (3.24), let

$$
\alpha_{\nu}^{*}(x)=\min \left\{\alpha_{i}(x): i \epsilon I(\nu)\right\}
$$

then (1.3) for the case (3.35) can be written

$$
\min _{x} \min _{Q} \sum_{\nu} \alpha_{\nu}^{*}(x) Q_{\nu}
$$

If for example $f$ is Cobb-Douglas with parameters $a_{\nu}$, the resultant pure location problem has in analogy with (3.4) the objective function

$$
\phi_{C D}^{*}(x)=\sum_{\nu} a_{\nu} \log \left[\alpha_{\nu}^{*}(x)\right]
$$

while if $f$ is $C E S$ with parameters $b_{v}$ and $c$, the result is

$$
\phi_{C E S}^{*}(x)=\sum_{\nu} b_{\nu}^{1-d}\left[\alpha_{\nu}^{*}(x)\right]^{d}
$$

where $d=c /(c+1)$, analogous with (3.6). Under the plausible assumption (in the present context) that $\left\{t_{i}: i \epsilon I(\nu)\right\}$ has a single member $t_{\nu},(3.37)$ and (3.38) can be replaced by

$$
\begin{gathered}
\phi_{C D}^{*}(x)=\sum_{\nu} a_{\nu} \log \left[\min _{i \in I(\nu)}\left\{\rho_{i}+\pi_{i}\right\}\right], \\
\phi_{C E S}^{*}(x)=\sum_{\nu} c_{\nu}\left[\min _{i \in I(\nu)}\left\{\rho_{i}+\pi_{i}\right\}\right]^{d}
\end{gathered}
$$

where $c_{\nu}=b_{\nu}^{1-d} t_{\nu}^{d}$ and $\pi_{i}=p_{i} / t_{i}$. Note that under the further assumption that $\left\{p_{i}: i \epsilon I(\nu)\right\}$ has a single member $p_{\nu}$,

$$
\min _{i \in I(\nu)}\left\{\rho_{i}+\pi_{i}\right\}=\left[\min _{i \in I(\nu)} \rho_{i}\right]+\pi_{\nu}
$$

with $\pi_{\nu}=p_{\nu} / t_{\nu}$

\section{Iterative Solution Methods}

The pure location problems obtained in section 3, by working out several cases of "explicit separation", require the minimization of fairly complex nonconvex functions $\phi(x)$. Since such problems are computationally nontrivial, it seems useful to present a class of iterative solution methods which are simple in concept and simple to program. These algorithms, which are based on characterizing an optimal location as a fixed point of an associated transformation of $R^{n}$, are presented in the present section, while computational experience with them will be reported in Part II.

As noted in (3.7) and (3.8), several of these problems have an objective function of the form

$$
\phi(x)=\sum_{1}^{m} \phi_{i}\left(\rho_{i}\right)
$$

where the functions $\phi_{i}(u)$ are twice differentiable for nonnegative arguments, and satisfy

$$
\phi_{i}^{\prime}>0 \quad(i=1,2, \ldots, m) .
$$


At any point $\bar{x}$ not an $s_{i}$, one has

$$
\operatorname{grad} \phi=\sum_{1}^{m} \phi_{i}^{\prime}\left(\rho_{i}\right)\left(\bar{x}-s_{i}\right) / \rho_{i}
$$

If $\bar{x}$ is to be a local minimum, then grad $\phi=0$ must hold, or equivalently

$$
\bar{x}=\left[\sum_{1}^{m} s_{i} \phi_{i}^{\prime}\left(\rho_{i}\right) / \rho_{i}\right] /\left[\sum_{1} \phi_{i}^{\prime}\left(\rho_{i}\right) / \rho_{i}\right],
$$

a formula which displays $\bar{x}$ as a fixed point of the function on the right-hand side, and incidentally as lying in the convex hull of the points $s_{i}$. This formula suggests the iterative scheme

$$
x^{(k+1)}=\left[\sum_{1}^{m} s_{i} \phi_{i}^{\prime}\left(\rho_{i}^{(k)}\right) / \rho_{i}^{(k)}\right] /\left[\sum_{1}^{m} \phi_{i}^{\prime}\left(\rho_{i}^{(k)}\right) / \rho_{i}^{(k)}\right] .
$$

Because of the presence of (in general, uncancelled) denominators $\rho_{i}^{(k)}$, this form can be unsuitable for numerical work when $x^{(k)}$ is near some $s_{i}$, say $s_{j}$, and should be replaced by the algebraically equivalent form obtained by multiplying numerator and denominator through by $\rho_{j}^{(k)}$. (This alternative form also shows that each $s_{j}$ is a fixed point of the transformation given by (4.5).) Of course, the test (3.16) for a local minimum at $s_{j}$ should be applied in such cases.

For the ordinary Weber problem, with objective function $\phi_{W}$ given by (1.1), the algorithm reads

$$
x^{(k+1)}=\left[\sum_{1}^{m} s_{i} t_{i} q_{i} / \rho_{i}^{(k)}\right] /\left[\sum_{1}^{m} t_{i} q_{i} / \rho_{i}^{(k)}\right] .
$$

This iterative scheme, which has been repeatedly rediscovered (e.g. [7], [9-11]), goes back at least as far as Weiszfeld [12], who also gave a convergence proof; the rapidity of that convergence has been confirmed in a number of instances, e.g. [13].

For the Cobb-Douglas case, with objective function $\phi_{C D}$ given by (3.4), the algorithm reads

$$
x^{(k+1)}=\left[\sum_{1}^{m} s_{i} a_{i} /\left(\rho_{i}^{(k)}+\pi_{i}\right) \rho_{i}^{(k)}\right] /\left[\sum_{1}^{m} a_{i} /\left(\rho_{i}^{(k)}+\pi_{i}\right) \rho_{i}^{(k)}\right],
$$

while for the CES case, with objective function $\phi_{C E S}$ given by (3.6), it reads

$$
x^{(k+1)}=\left[\sum_{1}^{m} s_{i} c_{i} /\left(\rho_{i}^{(k)}+\pi_{i}\right)^{1-d} \rho_{i}^{(k)}\right] /\left[\sum_{1}^{m} c_{i} /\left(\rho_{i}^{(k)}+\pi_{i}\right)^{1-d} \rho_{i}^{(k)}\right]
$$

This scheme (4.8) was considered by Cooper [14] for the case of all $\pi_{i}=0$, a limiting case of the situations of interest here. Note that if some $\pi_{j}=0$, and if the algorithm leads to an $x^{(k)}$ near $s_{j}$, then the numerator and denominator need to be multiplied by $\left[\rho_{j}^{(k)}\right]^{2}$ in $(4.7)$, and $\left[\rho_{j}^{(k)}\right]^{2-d}$ in (4.8), not just $\rho_{j}^{(k)}$. Note also that if some $\pi_{j}=0$, then for both $\phi_{C D}$ and $\phi_{C E S}$ the test for a local minimum at $s_{j}$ yields $A=\infty$ in (3.13) and thus an affirmative result for the test; for $\phi_{C E S}$ with all $\pi_{j}=0$, the fact that each $s_{j}$ yields a local minimum was observed by Cooper [15].

By the local convergence property $(L C P)$ for the pure location problem, we shall mean that each strict local minimum $\bar{x}$ of $\phi$, other than the points $s_{i}$, has a neighborhood $N(\bar{x})$ such that if the iterative process enters $N(\bar{x})$ at some stage, then it subsequently converges to $\bar{x}$ (in fact, in an-leastgeometric fashion). In a paper [16] dealing with the general scheme (4.5), Katz (op. cit., Theorem 4) shows that the LCP holds if, in addition to (4.2), the functions $\phi_{i}$ satisfy

$$
\phi_{i}^{\prime \prime}(u) \leqslant(3-n) \phi_{i}^{\prime}(u) / u \text {. }
$$


For both $\phi_{C D}$ and $\phi_{C E S}$ one has $\phi_{i}^{\prime \prime}<0$, so that for the low dimensions $(n \leqslant 3)$ of greatest practical interest, (4.9) is satisfied and hence the LCP is assured.

The objective functions (3.20), (3.21), (3.25), (3.27) and (3.30) have the more general form (3.31),

$$
\phi(x)=\sum_{\nu} \phi_{\nu}\left(\rho_{\nu}\right)
$$

with $\rho_{\nu}$ the vector with components $\left\{\rho_{i}: i \epsilon I(\nu)\right\}$. The twice-differentiable positive-valued functions $\phi_{\nu}\left(u_{\nu}\right)$, where $u_{\nu}$ is a vector of nonnegative variables $\left\{u_{i}: i \epsilon I(\nu)\right\}$, satisfy in all these cases the analog

$$
\partial \phi_{\nu} / \partial u_{i}>0 \quad(\text { all } i \epsilon I(\nu))
$$

of (4.2). The analysis by Katz [16] can be mimicked to obtain a generalization of (4.9) which, together with (4.11), is sufficient to assure the $L C P$ for the generalization (given later, below) of (4.5).

The details of this imitative analysis are straightforward by reference to [16], and therefore will not be repeated here. The result is that the condition

$$
\sum_{\nu}\left\{(n-3) \sum_{i \in I(\nu)}\left(1 / \rho_{i}\right)\left[\partial \phi_{\nu} / \partial u_{i}\right]\left(\rho_{\nu}\right)+\sum_{i, j \in I(\nu)}\left[\left(\bar{x}-s_{i}, \bar{x}-s_{j}\right) / \rho_{i} \rho_{j}\right]\left[\partial^{2} \phi_{\nu} / \partial u_{i} \partial u_{j}\right]\left(\rho_{\nu}\right)\right\} \leqslant 0,
$$

together with (4.11), sufficies for local convergence at $\bar{x}$. It follows that the conditions

$$
(n-3) \sum_{i \in I(\nu)}\left(1 / \rho_{i}\right)\left[\partial \phi_{\nu} / \partial u_{i}\right]\left(\rho_{\nu}\right)+\sum_{i, j \in I(\nu)}\left[\left(\bar{x}-s_{i}, \bar{x}-s_{j}\right) / \rho_{i} \rho_{j}\right]\left[\partial^{2} \phi_{\nu} / \partial u_{i} \partial u_{j}\right]\left(\rho_{\nu}\right) \leqslant 0
$$

for all $\nu$, together with (4.11), are sufficient. In particular, if for each $\nu$ the local minimum $\bar{x}$ lies outside the convex hull of the points $\left\{s_{i}: i \epsilon I(\nu)\right\}$, so that in (4.13) each scalar product $\left(\bar{x}-s_{i}\right.$, $\left.\bar{x}-s_{j}\right)>0$, and if each $\phi_{\nu}$ has all $\partial^{2} \phi_{\nu} / \partial u_{i} \partial u_{j} \leqslant 0$, and if $n \leqslant 3$, then local convergence holds at $\bar{x}$. For a more useful condition, one can employ the consequence

$$
\left(\bar{x}-s_{i}, \bar{x}-s_{j}\right) / \rho_{i} \rho_{j} \geqslant(-1)
$$

of the Cauchy-Schwartz inequality. If each $\phi_{v}$ satisfies

$$
\partial^{2} \phi_{\nu} / \partial u_{i} \partial u_{j} \leqslant 0 \quad(i, j \in I(\nu) ; i \neq j),
$$

then it follows from (4.13) and (4.14) that

$$
\sum_{i \in I(\nu)}\left\{(n-3)\left(1 / u_{i}\right) \partial \phi_{\nu} / \partial u_{i}+\partial^{2} \phi_{\nu} / \partial u_{i}^{2}\right\}-\sum\left\{\partial^{2} \phi_{\nu} / \partial u_{i} \partial u_{j}: i, j \epsilon I(\nu) ; i \neq j\right\} \leqslant 0,
$$

together with (4.11), is sufficient to assure that LCP. Note that (4.16) is a generalization of (4.9).

Consider first the objective functions $\phi_{C D}^{L}$ and $\phi_{C E S}^{L}$ of (3.20) and (3.21). For each of them, $\phi_{\nu}\left(u_{v}\right)$ has the form

$$
\phi_{\nu}\left(u_{\nu}\right)=g_{\nu}\left(\sum_{i \in I(\nu)} L_{i}\left\{u_{i}+\pi_{i}\right\}\right)
$$

so that (4.16) takes the form

$$
\sum_{i \in I(\nu)}\left\{(n-3)\left(L_{i} / u_{i}\right) g_{\nu}^{\prime}+L_{i}^{2} g_{\nu}^{\prime \prime}\right\}-\sum\left\{L_{i} L_{j} g_{\nu}^{\prime \prime}: i, j \epsilon I(\nu), i \neq j\right\} \leqslant 0
$$

with $g_{\nu}^{\prime}$ and $g_{\nu}^{\prime \prime}$ evaluated at the $g_{\nu}$-argument of (4.17). For (3.21), with $g_{\nu}(v)=v^{d}$, this condition is

$$
\sum_{i \in I(\nu)}\left\{(n-3)\left(L_{i} / u_{i}\right) \sum_{j \in I(\nu)} L_{j}\left(u_{j}+\pi_{j}\right)-(1-d) L_{i}^{2}\right\}+(1-d) \sum\left\{L_{i} L_{j}: i, j \in I(\nu) ; i \neq j\right\} \leqslant 0,
$$


or equivalently

$$
\begin{aligned}
\sum_{i \in I(\nu)} L_{i}^{2}\{ & \left.(n-3)\left(1 / u_{i}\right)\left(u_{i}+\pi_{i}\right)-(1-d)\right\} \\
& +\sum\left\{L_{i} L_{j}\left[(n-3)\left\{\left(u_{j}+\pi_{j}\right) / u_{i}+\left(u_{i}+\pi_{i}\right) / u_{j}\right\}+2(1-d)\right]: i, j \in I(\nu), i<j\right\} \leqslant 0 .
\end{aligned}
$$

Since $d<1$, assuming $n \leqslant 3$ assures that the first sum is $<0$. The generic summand of the second sum, divided by $L_{i} L_{j}$, is (if $n \leqslant 3$ )

$$
\leqslant(n-3)\left[u_{j} / u_{i}+u_{i} / u_{j}\right]+2(1-d)
$$

applying to the first term the inequality $z+1 / z \geqslant 2$ for $z>0$, proves for $n \leqslant 3$ that the last displayed expression is

$$
\leqslant 2(n-3)+2(1-d)=2(n-2-d),
$$

which is negative for $n \leqslant 2$. Thus, for $\phi_{C E S}^{L}, L C P$ holds for the planar and one-dimensional cases. The same argument, with $d=0$ in the later steps, yields the same conclusion for $\phi_{C D}^{L}$.

For the objective functions $\phi_{C D}^{C E S}$ and $\phi_{C E S}^{C E S}$ of (3.25) and (3.27), we have the generalization

$$
\phi_{\nu}\left(u_{\nu}\right)=g_{\nu}\left(\sum_{i \in I(\nu)} L_{i}\left\{u_{i}+\pi_{i}\right\}^{d_{\nu}}\right)
$$

of (4.17). Thus (4.16) takes the form

$$
\begin{aligned}
\sum_{i \in I(\nu)} L_{i}\left(u_{i}+\pi_{i}\right)^{d_{\nu}-2}\left[\left\{(n-3)\left(u_{i}+\pi_{i}\right) / u_{i}-\left(1-d_{\nu}\right) g_{\nu}^{\prime}+L_{i}\left(u_{i}+\pi_{i}\right)^{d}{ }_{\nu} g_{\nu}^{\prime \prime} d_{\nu}\right]\right. & \\
& -d_{\nu} \sum\left\{L_{i} L_{j}\left(u_{i}+\pi_{i}\right)^{d_{\nu}-1}\left(u_{j}+\pi_{j}\right)^{d}{ }_{\nu}^{-1} g_{\nu}^{\prime \prime}: i, j \in I(\nu) ; i \neq j\right\} \leqslant 0 .
\end{aligned}
$$

For (3.27), with $g_{\nu}(v)=v^{\delta}{ }_{\nu}$ where $\delta_{\nu}=d / d_{\nu}$, this yields

$$
\begin{aligned}
\sum_{i \in I(\nu)} L_{i}\left(u_{i}+\pi_{i}\right)^{d_{\nu}-2}\left[\left\{(n-3)\left(u_{i} \pi_{i}\right) / u_{i}-\left(1-d_{\nu}\right)\right\} \sum_{j \in I(\nu)} L_{j}\left(u_{j}+\pi_{j}\right)^{d_{\nu}}\right. \\
\left.\quad+L_{i}\left(u_{i}+\pi_{i}\right)^{d_{\nu}}\left(d-d_{\nu}\right)\right]-\left(d-d_{\nu}\right) \sum\left\{L_{i} L_{j}\left(u_{i}+\pi_{i}\right)^{d_{\nu}-1}\left(u_{j}+\pi_{j}\right)^{d}{ }_{\nu}{ }^{-1}: i, j \in I(\nu) ; i \neq j\right\} \leqslant 0
\end{aligned}
$$

or equivalently

$$
\begin{aligned}
& \sum_{i \in I(\nu)} L_{i}^{2}\left(u_{i}+\pi_{i}\right)^{2 d_{\nu}-2}\left[(n-3)\left(u_{i}+\pi_{i}\right) / u_{i}-\left(1-d_{\nu}\right)+\left(d-d_{\nu}\right)\right] \\
+ & \sum\left\{L _ { i } L _ { j } ( u _ { i } + \pi _ { i } ) ^ { d _ { \nu } - 1 } ( u _ { j } + \pi _ { j } ) ^ { d _ { \nu } - 1 } \left[\left\{(n-3)\left(u_{i}+\pi_{i}\right) / u_{i}-\left(1-d_{\nu}\right)\right\}\left(u_{j}+\pi_{j}\right)^{2}\right.\right. \\
+ & \left.\left.\left\{(n-3)\left(u_{j}+\pi_{j}\right) / u_{j}-\left(1-d_{\nu}\right)\right\}\left(u_{i}+\pi_{i}\right)^{2}-2\left(d-d_{\nu}\right)\left(u_{i}+\pi_{i}\right)\left(u_{j}+\pi_{j}\right)\right]\right\} \\
& : i, j \in I(\nu) ; i<j\} \leqslant 0 .
\end{aligned}
$$

Since $d<1$, the first sum is negative for $n \leqslant 3$. As for the second sum, the factor [---] in its generic summand is for $n \leqslant 3$

$$
\begin{aligned}
& \leqslant-\left[\left\{\left(1-d_{\nu}\right)-(n-3)\right\}\left\{\left(u_{i}+\pi_{i}\right)^{2}+\left(u_{j}+\pi_{j}\right)^{2}\right\}+2\left(d-d_{\nu}\right)\left(u_{i}+\pi_{i}\right)\left(u_{j}+\pi_{j}\right)\right] \\
& =-\left\{\left(1-d_{\nu}\right)-(n-3)\right\} Q\left(u_{i}+\pi_{i}, u_{j}+\pi_{j}\right),
\end{aligned}
$$

where the coefficient $\left(1-d_{\nu}\right)-(n-3)$ is positive for $n \leqslant 3$, and the quadratic form $Q$ is given by

$$
Q(v, w)=v^{2}+w^{2}+2 k v w, \quad k=\left(d-d_{\nu}\right) /\left\{\left(1-d_{\nu}\right)-(n-3)\right\} .
$$

$Q$ is positive definite for $k^{2}<1$. Since $k<1$ follows from the fact that $d<1$, it suffices to have 
$k>(-1)$. For $n=3$ this is true if $d+1>2 d_{\nu}$, while for $n \leqslant 2$ it follows without additional restriction. For (3.25) the analysis is similar, corresponding to $d=0$.

For the objective function $\phi_{C E S}^{C D}$ of (3.30), condition (4.16) leads to

$$
\begin{gathered}
\sum_{i \in I(\nu)} a_{i}^{\prime}\left(u_{i}+\pi_{i}\right)^{-2}\left\{(n-3)\left(u_{i}+\pi_{i}\right) / u_{i}-1\right\} \\
-\sum\left\{a_{i}^{\prime} a_{j}^{\prime} /\left(u_{i}+\pi_{i}\right)\left(u_{j}+\pi_{j}\right): i, j \epsilon I(\nu) ; i \neq j\right\} \leqslant 0,
\end{gathered}
$$

which holds for $n \leqslant 3$.

The generalization of (4.5) to the situation (4.7), for which the preceding convergence analyses employing (4.16) were given, is obtained using the generalization

$$
\operatorname{grad} \phi=\sum_{\nu} \sum_{i \in I(\nu)}\left[\partial \phi_{\nu} / \partial u_{i}\right]\left(\rho_{\nu}\right)\left(\bar{x}-s_{i}\right) / \rho_{i}
$$

of (4.3). The iterative scheme is

$$
\begin{aligned}
x^{(k+1)} & =\left[\sum_{\nu} \sum_{i \in I(\nu)} s_{i}\left[\partial \phi_{\nu} / \partial u_{i}\right]\left(\rho_{\nu}\right) / \rho_{i}\right] \\
& \div\left[\sum_{\nu} \sum_{i \in I(\nu)}\left[\partial \phi_{\nu} / \partial u_{i}\right]\left(\rho_{\nu}\right) / \rho_{i}\right] .
\end{aligned}
$$

For $x^{(k)}$ near some $s_{i}$, precautions like those noted under (4.3) are required.

It remains to consider objective functions like the $\phi_{C D}^{*}$ and $\phi_{C E S}^{*}$ of (3.37) and (3.38), whose general form is

$$
\phi^{*}(x)=\sum_{\nu} \phi_{\nu}\left[\alpha_{\nu}^{*}(x)\right], \quad \alpha_{\nu}^{*}(x)=\min \left\{\alpha_{i}(x): i \epsilon I(\nu)\right\}
$$

with $\alpha_{i}$ defined by (3.24). Let us call $x$ exceptional if a tie occurs in the definition of some $\alpha_{\nu}^{*}(x)$; for any nonexceptional point $x$, let $i(\nu, x) \epsilon I(\nu)$ be the unique index for which the minimum occurs. For nonexceptional points, the previous analyses can be carried over by replacing $\alpha_{\nu}^{*}(x)$ with $\alpha_{i(\nu, x)}(x)$. The reason is that these analyses-testing for a local minimum at a point $s_{i}$, or for local convergence to a local minimum (not an $s_{i}$ ) of an iterative scheme based on a zero gradient (itself a local construct) - deal only with local behavior of $\phi^{*}$, and each nonexceptional point $x$ has a neighborhood consisting entirely of nonexceptional points $y$ for which $i(\nu, y)=i(\nu, x)$ for all $\nu$. But the "radius of convergence" around a local minimum is reduced by the need to avoid contact with the set of exceptional points; a local minimum lying near this set may therefore be "hard to get at" for the algorithm. If the algorithm generates an $x^{(k)}$ which is an exceptional point, it is natural to proceed by breaking the tie arbitrarily, and the effect of this seems difficult to predict. (An alternative is to employ a more complex logic involving "branching" when an exceptional $x^{(k)}$ is encountered.) The ability to detect a minimizing point which is exceptional is a priori dubious. These problems are explored on an empirical basis in some of the computational experiments to be reported in Part II.

\section{References}

[1] Sakashita, N., Production function, demand function and location theory of the firm, Papers of Reg. Sci. Assoc. 20, 109-122 (1967).

[2] Wendell, R. E., and Hurter, A. P., Optimal locations on a network, Trans. Sci. 7, 18-33 (1973).

[3] Hurter, A. P., and Wendell, R. E., Location and production-A special case, J. Reg. Sci. 12, 243-247 (1972).

[4] Cobb, C. W., and Douglas, P. H., A theory of production, Amer. Eco. Rev. (Papers \& Proceedings) 18, 139-205 (1926).

[5] Arrow, K., Chenery, H., Minhas, B. S., and Solow, R. M., Capital-labor substitution and economic efficiency. Rev. Eco. \& Statistics 63, 225-250 (1961).

[6] Shephard, R. W., Theory of Cost and Production Functions, Princeton Univ. Press, Princeton, N.J., 1970. 
[7] Kuhn, H. W., and Kuenne, R. E., An efficient algorithm for the numerical solution of the generalized Weber problem in spatial economics, J. Reg. Sci. 4, 21-33 (1962).

[8] Uzawa, H., Production functions with constant elasticities of substitution, Rev. Eco. Studies 30, (1962).

[9] Miehle, W., Link-length minimization in networks, Oper. Res. 6, 232-243 (1958).

[10] Palermo, F. P., A network minimization problem, IBM J. Res. \& Dev. 5, 335-337 (1961).

[11] Cooper, L., Location-allocation problems, Oper. Res. 11, 331-343 (1963).

[12] Weiszfeld, E., Sur le point pour lequel la somme des distances de n points donnes est minimum, Tohuku Math. J. 43, 355-386 (1936).

[13] Jordan, R. H., et al., Systems Analysis of Inland Consolidation Centers for Marine Cargo, Nat. Bur. Stand. (U.S.), Tech. Note 530, 162 pages (Nov. 1970).

[14] Cooper, L., Solution of generalized locational equilibrium problems, J. Reg. Sci. 7, 1-18 (1967).

[15] Cooper, L., An extension of the generalized Weber problem, J. Reg. Sci. 8, 181-197 (1968).

[16] Katz, I. N., On the convergence of a numerical scheme for solving some locational equilibrium problems, SIAM J. Appl. Math. 17, 1224-1231 (1969).

(Paper 78B2-403) 